Cinémas

Revue d'études cinématographiques

Journal of Film Studies

\title{
Qu'est-ce que tu as vu à Chelmno? Shoah, un monumentaire
}

\section{Jean-Pierre Esquenazi}

Volume 12, numéro 1, automne 2001

Le Paysage au cinéma

URI : https://id.erudit.org/iderudit/024872ar

DOI : https://doi.org/10.7202/024872ar

Aller au sommaire du numéro

Éditeur(s)

Cinémas

ISSN

1181-6945 (imprimé)

1705-6500 (numérique)

Découvrir la revue

Citer cet article

Esquenazi, J.-P. (2001). Qu'est-ce que tu as vu à Chelmno? Shoah, un monumentaire. Cinémas, 12(1), 147-165. https://doi.org/10.7202/024872ar

\section{Résumé de l'article}

Shoah pose un problème à la théorie du documentaire, dans la mesure où le film ne se présente pas comme l'enregistrement du réel par un Je-origine (lui-même réel). À l'inverse de ce qu'opère un " documentariste classique ", l'auteur montre que le film se présente comme constituant sa référence, non son enregistrement. Désignant ce film par le terme de " monumentaire ", l'auteur étudie les processus référentiels qui y sont engagés à la lumière de Les Individus de P.F. Strawson. 


\title{
Qu'est-ce que tu as vu à Chelmno? Shoah, un monumentaire
}

\section{Jean-Pierre Esquenazi}

\begin{abstract}
RÉSUMÉ
Shoah pose un problème à la théorie du documentaire, dans la mesure où le film ne se présente pas comme l'enregistrement du réel par un Je-origine (lui-même réel). À l'inverse de ce qu'opère un "documentariste classique", l'auteur montre que le film se présente comme constituant sa référence, non son enregistrement. Désignant ce film par le terme de "monumentaire", l'auteur étudie les processus référentiels qui y sont engagés à la lumière de Les Individus de P.F. Strawson.
\end{abstract}

\section{ABSTRACT}

The film Shoah poses a problem for the theory of documentary cinema: it does not present itself as a transcription of reality by an equally real "I" at its source. The filmmaker reveals that the film constitutes his reference rather than recording him directly, the way a classical documentary would. Calling such a film "monumentary," the author analyses the referential processes at work in it, following the ideas set out by P.F. Strawson in his work Individuals.

\section{Présentation}

Généralement, les documentaires sont présentés comme des films qui ont, d'une façon ou d'une autre, "enregistré le réel». De même, le spectateur présuppose au documentaire un $J e-$ origine réel, autrement dit un cinéaste physiquement confronté à la réalité qui est l'objet même du film (Odin, 1984, p. 263- 
278). À cet égard, Shoah de Claude Lanzman pose un problème théorique intéressant, puisque l'auteur s'interdit au préalable l'accès à la réalité même du film. Une double question se pose alors: dans quelle mesure peut-on parler de documentaire à propos de Shoah? Quel est éventuellement son mode spécifique de présentation? Le présent article - à propos duquel l'auteur s'excuse par avance d'avoir délaissé les nombreux textes écrits sur le film - va plutôt s'articuler autour de ce problème précis posé par Shoah.

Plusieurs termes seront ici utilisés de manière assez singulière. Pour éviter certains malentendus, nous voulons cependant apporter quelques précisions. Nous supposerons qu'un film documentaire se distingue d'un film de fiction en ce qu'il fait explicitement référence à un "quelque chose " présumé réel: dans le discours du film, cette opération de référenciation est corroborée par le dispositif de prise de vues. Nous ne nous attarderons pas sur cette ébauche de définition, par ailleurs largement reconnue. Nous désignerons ce "quelque chose" par objet. Nous parlerons du "Sujet» du film comme du sujet d'un énoncé: le Sujet du film serait la personne dont l'énoncé dessine la figure, et qui serait à l'origine des énoncés '.

L'essentiel de la discussion portera sur l'objet du film. Nous soutiendrons que le style de Shoah établit un rapport particulier entre le discours du film et son objet de référence. Celui-ci n'est pas présupposé par le film, il devient son problème. Ce que le film interroge est la constitution de l'objet "Shoah" comme une référence réelle, et le sens donné au mot « réel »? Selon nous, cette question est précisément ce qui situe Shoah dans les marges du film documentaire. En effet, au sein de cette catégorie de films, la référence est communément présupposée, reconnue a priori. Bien sûr, les documentaristes pratiquent toujours, revendiquent parfois, des modes différents d'écriture, afin de produire des "points de vue documentés" sur leurs objets. Néanmoins, la réalité - la "vie" dirait Vertov - reste pour eux la source où ils puisent constamment.

Ainsi, l'enjeu implicite de Shoah serait d'approcher une définition de la réalité. Celle-ci serait-elle un ordre qu'il faut représenter, interpréter, ou encore une série d'événements dont il faut 
organiser les contours et cerner les conséquences? Après avoir identifié la réponse suggérée par le film, il s'agira ici de préparer un terrain propice à une réflexion sur le statut de la réalité. Ce développement se déroulera en quatre étapes. D’abord, il y aura lieu d'analyser Shoah, afin de mieux comprendre de quelle manière la Solution finale constitue son objet. Dans un deuxième temps, en confrontant le style de Shoah à une œuvre de forme "classique", le problème de l'acte de référence sera pris en considération. Ensuite, il s'agira de montrer comment le choix d'un mode de référence peut déterminer une esthétique cinématographique de la perception. Dès maintenant, l'option choisie par Lanzman dans Shoah sera désignée comme une "esthétique de la constitution». Enfin, une attention particulière sera accordée à certains traits des films relevant de cette esthétique (les statuts du Sujet, de l'objet et de la temporalité).

\section{Construction de Shoah}

La première scène de Shoah confronte la problématique du film à l'intensité émotionnelle qu'elle dégage. Introduit par un long panneau, le film relate l'histoire de Simon Srebnik, un des deux survivants du camp de Chelmno, sauvé par la beauté de sa voix et la chance d'avoir juste été étourdi par la balle qui lui était destinée. Contacté par Lanzman, Simon Srebnik accepte de revenir à Chelmno.

La première image du film montre une barque qui s'éloigne: un homme, de dos, pousse une perche, tandis qu'un homme, assis à l'autre extrémité de l'embarcadère, entonne les premiers couplets d'une chanson. Il pleut. Au bout d'un court instant, une voix off se fait entendre. Les mots - dits en polonais, et bientôt traduits en français - confient: "Il avait treize ans et demi, il avair une belle voix, et on l'entendait..." Le film présente Simon Srebnik comme un «il»: «il est celui qui chantait..." Nous l'observons à distance, alors qu'il revient vers le passé.

Dans le plan rapproché, consécutif, Simon Srebnik poursuit sa chanson. Depuis la berge, un homme l'observe. Puis, le chant s'arrête. Les yeux de Simon scrutent les alentours. Face à la caméra, en plan rapproché, il est maintenant sur un chemin, 
dans un bois. Il amorce quelques hochements de la tête, puis ralentit. Après quarante-cinq secondes, il interrompt sa marche pour porter progressivement son regard vers l'avant. De profil, toujours en plan rapproché, il déclare: "Difficile à reconnaître, mais c'était ici... Ici on brûlait les gens. Beaucoup de gens ont été brûlés ici... Oui, c'est le lieu.» Son regard est toujours en éveil, tandis que l'image tremble. Sa marche, son regard et sa parole commandent désormais le film. Nous le suivons une fois qu'il est entré dans le camp.

Après quarante secondes, un lent panoramique ouvre sur une clairière ordonnée par des murs en pierre, bas et rectangulaires. Le sol est couvert d'une herbe coupée à ras. Après une trentaine de secondes de silence, on entend la voix de Simon: «Personne n'en repartait jamais." Par ce plan subjectif, nous découvrons ce que Simon Srebnik voit. Nous entrevoyons ıne clairière, des enceintes en pierre, basses et rectangulaires, tandis que lui "voit" le camp. Plus exactement, il le "visionne", car ce dont il parle n'est pas accessible au spectateur.

Cadré alors en plan rapproché, Simon Srebnik poursuit : «Les camions à gaz arrivaient par là; il y avait d'immenses fours... Et ensuite on jetait les corps dans ces fours, et les flammes montaient jusqu'au ciel.» La voix off de Lanzman s'étonne: "Jusqu'au ciel? "Simon Srebnik reprend: "Oui. C'était terrible. " L'insistance de Lanzman désigne notre impuissance: nous ne pouvons pas voir, fût-ce imaginer, ces flammes qui montent jusqu'au ciel. Ce qui s'est passé ici n'a pas de commune mesure avec ce que nous pourrions imaginer.

La séquence a commencé depuis à peine cinq minutes. Nous suivons maintenant Simon Srebnik, qui savance dans la clairière; il est de dos, puis de profil en plan rapproché. Nous découvrons Lanzman qui marche à ses côtés. Simon Srebnik prend à nouveau la parole: "On ne peut pas raconter cela... Personne ne peut se représenter ce qui s’est passé ici. Impossible... Et personne ne peut comprendre cela... et moi-même aujourd'hui..." Sa main s'élève jusqu'à ses yeux pour accompagner sa parole. Il apparait maintenant seul, au loin, entouré par les rectangles des murs en pierre. On l'entend encore: «... Je ne crois pas que je suis ici... C'était toujours tranquille ici. Toujours. Quand on 
brûlait chaque jour deux mille personnes, des Juifs, c'était également tranquille. Personne ne criait. Chacun faisait son travail. C'était silencieux, paisible. Comme maintenant.»

Pour Simon Srebnik, toute intention de dire la Shoah est irrémédiablement vouée à l'échec. Quels mots ou quelles images pourraient dire ce qui ne correspond à rien d'autre dans l'histoire? Les traces visuelles ou sonores de la Solution finale ont disparu. La séquence parait désigner son propre objet comme un objet inaccessible. La Shoah ne serait pas désignable en ces termes: "Voici la Shoah." Lobjet même du film ne serait pas une matière première à explorer, pour laquelle il s'agirait d'avoir un point de vue.

Qu'en est-il alors du projet d'un film documentaire dont l'objet se dérobe? S'agit-il de dévoiler cet objet, de lui donner une certaine consistance ou de déterminer sa forme originelle? Le film n'a pas pour souci de relever les qualités de cet objet, mais bien de le constituer. À cet effet, le film met en œuvre deux procédures dès la première séquence. D'une part, il s'agit de localiser les lieux de la Solution finale. Simon Srebnik a dit: «Beaucoup de gens ont brûlé ici... Oui c'est le lieu!" Définir précisément les limites où "çà a eu lieu, là réside la première ambition du film. Ensuite, il y a la parole des témoins qui ont survécu aux camps. Simon Srebnik a vu et entendu ce qui est pour nous invisible et inaudible. Il a vu les flammes qui montaient jusqu'au ciel; la parole des survivants contient de telles visions: les chambres à gaz, les fours crématoires, les corps réduits en cendres sont dans la voix de ceux qui ont survécu. Les règles, les déplacements, le rythme de la vie dans les camps peuvent ressurgir. Le film doit alors entrainer ces survivants - du moins leur esprit - dans l'enceinte des camps, afin de rendre possible le retour vers ce passé. Les survivants pourront décrire la mise en ouvre de la Solution finale, et le jeu de langage qui l'a organisée.

Voir Shoah, c'est d'abord tracer des lignes sur une terre anonyme, puis visionner des paroles qui correspondent aux lieux délimités par ces lignes.

Le film confronte ainsi deux séries d'images sonores. La première série, ici désignée par l'Arpentage, peut être conçue comme un vaste mouvement de délimitation et de définition de 
bornes, de frontières et de contextes. Dans une séquence où il interroge Jan Piwonski, aiguilleur à Sobibor, Lanzman lui demande de tracer la limite exacte de l'entrée du camp. Après quelques pas, il s'exprime: "Si je suis ici, je suis dans l'enceinte du camp?» Il revient deux mètres en arrière et insiste: «Et si je suis là, à 15 mètres de la gare, je suis à l'extérieur du camp? La partie polonaise et la mort? " Il interroge tous ceux qui étaient à la frontière, pour savoir ce qu'ils pouvaient voir ou entendre. Le film est un acte qui trace, marque, balise, les frontières au-delà desquelles le monde changeait. Shoah explore les effets d'une proximité, les silences et les mensonges. La caméra est mouvante: elle accompagne les déplacements impromptus de Lanzman. Fidèle aux indications recueillies, il fait émerger les domaines que s'était appropriée la Solution finale. La caméra, en attente, observe l'interrogateur et l'interrogé, puis, après une indication ou une description, désigne les partages, le contour, la transformation d'un lieu, et le signe d'une limite temporelle (une synagogue abandonnée, un cimetière juif en ruine).

La seconde série enregistre la parole des survivants: elle est l'Invocation. L'un des survivants décrit d'une voix mesurée, orientée vers le hors-champ, ce qu'il a vu, entendu, senti, dans le camp où il a été enfermé. Nous écoutons la litanie des faits, égrenée par celui qui a été épargné. Le gros plan isole les visages, parfois en rendant totalement flou tout ce qui l'entoure, comme pour le récit de Rudolf Vrba, un survivant d'Auschwitz. Lanzman n'apparaît plus: il se place lui-même dans le hors-champ de cette série, où seuls apparaissent ceux qui ont été à l'intérieur des camps. Il se contente de faire revenir, effectivement ou en pensée, celui qui détient la parole dans les limites du camp. Il demande parfois une précision, et veille à ce que le survivant aille au bout de son témoignage. Ainsi, quand Abraham Bomba, le coiffeur de Treblinka, doit s'arrêter, bouleversé par ce qui l'agite, la voix de Lanzman exige: "You have to, Abe... I apologize... You must go on...» La caméra attend sans répit qu’Abraham Bomba reprenne son récit. Chacune de ces séquences est comme un rituel, une cérémonie où chaque Juif survivant est un officiant qui n'évoque pas sa vie, qui invoque un drame pour que celui-ci habite et hante le spectateur. 
Les récits des survivants s'élèvent, quittent le présent, pour remonter le temps: alors qu'un survivant s'exprime, la caméra en mouvement découvre la terre du camp. Elle décrit un trajet identique à celui de Philip Müller dans Auschwitz, quand celuici vit pour la première fois les fours crématoires. La caméra scrute également la terre de Chelmno, quand Mordechai Podchelbnik parle de l'enfouissement des gazés. Ce sont de longs parcours dans les lieux mêmes de l'Arpentage, conduits par une caméra toujours en mouvement. Comme le dirait Deleuze: «Ce qu'on voit, c'est uniquement la terre déserte, mais cette terre déserte est comme lourde de ce qu'il y a en dessous, qui est justement ce dont la voix nous parle... Et si la voix nous parle de cadavres, c'est toute la lignée des cadavres qui vient prendre place sous terre ${ }^{2}$." Ce n'est pourtant pas Shoah que Deleuze commente ici, mais sa description de l'art des Straub est assez proche du travail d'Invocation de Shoah, dans la mesure où le film parvient à nous faire "voir" les paroles des survivants, et à faire surgir l'épaisseur du sang enfoui dans les terres.

Le Sujet (ou: l'image du Sujet mise en scène par le film) se situe à la frontière de l'Arpentage et de l'Invocation. Dans l'Arpentage, le film expose l'âpreté de son cheminement près des lieux de la Solution finale, afin d'en tracer les limites: le Sujet se fait voir. Dans l'Invocation, le film conjoint une parole testimoniale et une terre gorgée de cadavres. Ici, le Sujet fait "voir" (en l'occurrence "visionner"). Par la place qu'il occupe tant à l'intérieur du film (l'Arpentage) que devant le film (l'Invocation), le Sujet est donc celui qui assure la transition, celui qui suture pour reprendre le terme de Jean-Paul Oudart dont nous avons montré la généralité dans notre ouvrage Film, perception et mémoire (Esquenazi, 1994).

\section{Modes de références}

Nous avons vu que le film récuse la possibilité d'introduire sous forme désignative sa référence : la Solution finale. Il y paraît impossible de tendre la main et de dire "Voici ce qu'était la Shoah". Plutôt que de m'étendre sur les origines de cette impossibilité, au centre de très nombreuses discussions, je vais plutôt m'attarder sur les implications discursives de ce déni. Pour 
mieux comprendre ce qui est mis en œuvre dans le film, il est bon, selon moi, de détailler la procédure que le film se refuse à employer. Celle-ci, assez commune pour opérer un acte de référence dans un discours, permet d'y introduire des objets réels, des "particuliers" selon la terminologie de la théorie de la référence du philosophe anglais Strawson. Référer sert alors à désigner l'objet réel par ce que Strawson appelle une «description identifiante" (1973, p. 34). Dans un contexte particulier, cette description suffit aux partenaires du discours pour identifier l'objet unique auquel il se réfere. Dans le cadre d'une telle description, l'introduction d'un objet présuppose une proposition empirique, un savoir plus ou moins précis sur cet objet: les partenaires doivent savoir — du moins, doivent-ils le croire - que les qualités nommées par la description identifiante s'appliquent à cet objet (dans un documentaire sur les pingouins, le spectateur doit savoir à quoi ressemble le pingouin pour accepter l'image qu'on lui propose d'un véritable pingouin). L'emploi d'une description identifiante présuppose la reconnaissance d'une certaine définition du monde.

Une seconde condition est indispensable pour qu'un tel acte de référence arrive à ses fins: l'interlocuteur doit croire que l'énonciateur est en mesure de vérifier la proposition présupposée. Il n'est pas évident d'admettre un acte de référence à la couleur bleue, accompli par un aveugle de naissance. Dans son texte "Film documentaire, lecture documentarisante", Roger Odin a admis cette seconde condition dans le cas du documentaire, pour en donner une description précise. Il montre qu'il y a lecture documentarisante (ce que nous appelons un "acte de référence réussi ») quand le spectateur "construit l'image de l'Énonciateur, en présupposant la réalité de cet Énonciateur" (Odin, 1984, p. 267).

Qu'en est-il de Shoah? D'abord, il y est dit que le jeu de langage qui permettrait de dire l'objet, de le dire comme un objet extérieur auquel pourraient référer avec succès des locuteurs, n'existe pas. Il n'y a plus de traces tangibles de la Solution finale (comme à Treblinka ou à Chelmno). À tout le moins, ces traces n'autorisent-elles aucune compréhension du fonctionnement des camps (comme à Auschwitz) : ce qui demeure visible corres- 
pond davantage à un masque ou à un écran entre l'objet (le camp, tel qu'il était entre 1942 et 1944) et notre regard. En ce sens, aucune description identifiante n'est possible, qu'elle soit imagée ou langagière. Ce n'est pas tant que l'objet n'existe pas, mais plutôt que le jeu de langage qui pourrait référer à l'objet fait défaut.

Le film ne réfere donc pas, en ce sens, à un objet nommé "Shoah». Il effectue, nous l'avons vu, deux actes qui consistent, d'une part, à tracer les limites géographiques de la Solution finale et, d'autre part, à spécifier de l'intérieur - à travers les récits des survivants - la Solution finale tel un jeu de langage, au sens large donné à cette expression par Wittgenstein : l'ensemble d'une activité et des règles qui ordonnent cette activité.

Ce que recouvre le dessein du film, eu égard aux propositions de Strawson, n'est pas clairement défini. Dans Les Individus, le philosophe met à jour un autre procédé qui vise à introduire des objets réels. Il ne s'agit plus, alors, d'introduire des objets particuliers, bien individualisés, mais plutôt ce qu'il nomme des concepts-traits: "La neige, l'eau, le charbon, et l'or, par exemple, ne sont pas des propriétés ou des caractéristiques de particuliers, mais des genres de substances en général - bien qu'être fait de neige ou être fait d'or soient eux des caractéristiques de particulier» (Strawson, 1973, p. 227). Ces concepts-traits trouvent place dans des énoncés qui-placent-des-traits comme: «il y a de la neige". D'après Strawson, l'usage que nous faisons du langage, plus particulièrement lorsqu'il s'agit des descriptions identifiantes, implique une aptitude à reconnaître et comprendre ces énoncés qui-placent-des-traits. Pour identifier un chat particulier, il est impératif de reconnaître au préalable la présence-dechat, et de disposer d'un énoncé comme "Il y a là $d u$ chat ":

En fait, le concept de trait-de-chat fournit une base pour l'idée de réidentification de chats particuliers. Car ce concept comprend l'idée d'une configuration caractéristique dans l'occupation de l'espace..., d'un cheminement continu tracé à travers l'espace-temps par une telle configuration caractéristique (Strawson, 1973, p. 233). 
Les présuppositions engagées par ces énoncés qui-placent-destraits ne concernent plus des propositions empiriques qualitatives, mais la possibilité de déterminer une certaine pratique de l'espace et du temps: constituer une configuration d'espacetemps. Dès lors, le travail du film revient à constituer le concepttrait Solution finale. Il sagit, ni plus ni moins, de donner un sens à l'expression "Il y a eu, à tel et tel endroit, la Shoah".

Cet acte est encore, nous semble-t-il, un acte de référence ou, plus exactement, un acte qui constitue la référence. Il vise à exprimer un énoncé comme "Il y a $d u$ tel-et-tel". Pour ce faire, il doit élaborer la configuration d'un espace et d'un temps, identifrable à la Solution finale. Nos deux séries d'images sonores, l'Arpentage et l'Invocation, prennent tout leur sens. L'Arpentage définit les limites de la configuration, et son influence sur les configurations voisines et les individus qui y vivent. L'Invocation précise l'organisation interne de la configuration, sa structure, les rôles de chaque partie; elle fournit des critères de distinction des parties, pour le dire à la manière de Strawson. Shoah est un documentaire qui construit sa propre référence. C'est en cela, nous semble-t-il, qu'il se distingue passablement des films documentaires.

Remarquons encore que la crédibilité de Shoah ne passe plus (ou pas seulement) par la construction d'un Énonciateur présupposé réel. Ce sont les survivants convoqués par le film qui en supportent la construction. Leurs témoignages déterminent le jugement des spectateurs: une fois qu'ils les acceptent, la configuration révélée par le film est stabilisée, et le concept-trait Solution finale peut référer à un objet réel.

\section{Qu'est-ce que voir?}

Glissant d'un style à l'autre pour introduire la référence, on passe également d'une conception de la perception à une autre. Nous examinerons maintenant cette différence, et les réserves implicites de chacune de ces conceptions l'une pour l'autre. Le spectateur de Shoah n'est pas seulement appelé à voir le film, mais doit également se figurer les «images" dont le film parle. Il est en un sens paradoxal qu'un tel film - a fortiori, un film appelant son spectateur à avoir des "visions», et considérant ses 
visions comme réelles - puisse être désigné comme un «documentaire". Cette dénomination peut éventuellement lui être contestée. Prenons acte de ce dernier jugement pour examiner ce qui le motive. Un tel refus tient à des affirmations comme: "On ne saurait voir que ce qui est déjà là. La perception s'exerce sur un ensemble déjà constitué. " De ce point de vue, la perception serait d'abord un enregistrement. À cet égard, il faut noter qu'une telle conception n'exclut pas l'implication d'un sujet qui réorganise les éléments de sa perception selon un "point de vue documenté» — ou le travail du «ciné-œil» — qui produit une connaissance non immédiatement apparente. Dans un documentaire "classique", qui s'accommode plutôt de cette conception, l'image d'un objet implique que cet objet est réel, c'est-à-dire qu'il existe un jeu de langage où cet objet est répertorié comme tel. Cette image fait référence à cet objet, au premier sens de ce terme précédemment mentionné. L'existence d'un monde réel où cet objet prend place est donc présupposée. Il s'agit alors pour le spectateur de reconnaître à l'image offerte au regard d'appartenir à ce monde réel.

D'après une telle compréhension de l'acte perceptif - autant dire une vision "classique" du film documentaire - s'opère avec force la distinction entre ce qui est dit des objets auxquels le discours fait référence et l'existence même de ces objets, présupposée indépendante du discours. Voir, ici, c'est donc prendre acte des objets présents dans le champ visuel, et proposer une interprétation de l'organisation de ce champ (qui reste lui-même présupposé).

Cette représentation de la perception et le jeu de langage qui lui est associé ne sont pas contradictoires, dans la mesure où des objets inconnus, non identifiés, ne sont pas rencontrés. Quand on découvre un objet inconnu, seule notre perception actuelle fournit des renseignements à son propos. Cet objet ne peut plus être considéré comme le particulier d'une série d'objets du même type, identifiable à cette série. Aucun monde réel connu ne vient à notre secours.

Shoah propose de rendre réel ce que nous visionnons. Parcourant la terre de Chelmno, la rampe de Treblinka ou l'usine à crémation d'Auschwitz, il demande à son spectateur de voir les 
cadavres qui sont jetés dans la terre gelée, les Juifs épuisés qui descendent du train, qui sont battus et conduits aux chambres à gaz, les membres des Sonderkommando qui poussent les cadavres dans les fours. Voir, c'est alors se situer soi-même dans le champ de vision, et être acteur de la vision. La perception n'est plus reconnaissance, elle est invention du monde vécu. Elle est un acte qui engage le corps tout entier, le sens et l'esprit, et qui constitue l'ensemble des configurations des concepts-traits, avant de dire "Il y a $d u$ ceci et $d u$ cela". De ce point de vue, le paradoxe se trouve plutôt dans la perception-reconnaissance, qui suppose un esprit et un œil travaillant séparément (quand le premier trie et nomme, le second constate une présence).

Parmi les objections à l'endroit de Shoah, l'attitude perceptive proposée par le film est souvent réduite à une démarche de circonstances. On a tendance à dire que Shoah-le film aurait dû effectuer ce travail de constitution, parce que Shoah-l'objet a disparu. Devant un objet consistant et présent, il serait inutile et néfaste de refuser ce constat d'existence. Mais n'est-il pas possible de considérer la découverte d'un objet - par exemple le château de Versailles - en tant qu'elle est une expérience, une expérimentation qui permette de décrire en quoi ce château est "réel " pour le Sujet de cette expérience? Il s'agit donc de rétorquer à ce type d'objection que l'existence n'est pas une propriété du monde, mais une caractéristique propre à certains jeux de langage. Shoah est une manière autre de dire le réel.

Une autre critique à l'égard de Shoah rappelle que le film nous parle du passé, et que les perceptions du passé ne sont rien d'autre que des visions de ce passé. Tout dépend de la perspective choisie: cette vision du passé peut être prise pour une reconstitution du passé, et les documents dont on dispose pour les éléments d'un puzzle, qui vont, in fine, reconstituer une figure identique à ce passé. Ici, une "figure idéale du passé " sert de constante, toujours présupposée, même si elle est à venir. La vision apparaît alors comme un substitut de l'enregistrement qui n'a pas été matériellement réalisé. À l'inverse, la vision du passé peut être organisée depuis ce passé: il s'agit alors de faire revivre un présent révolu dans un présent réactualisé, de faire sentir le retentissement (plus que l'écho) du passé dans le présent des 
personnes. Il me semble que De Nuremberg à Nuremberg, le film de Rossif, représente la première tendance, et Shoah la seconde.

De la sorte, il s'agit d'opposer systématiquement deux façons de voir, deux conceptions de l'acte perceptif. À cet effet, il est important de dire que cette opposition ne détermine pas un choix, mais plutôt des types de situations qui, l'une sans l'autre, seraient lacunaires. De même, pour Strawson, la référence utilisant des descriptions identifiantes et les énoncés qui-placent-des-traits sont complémentaires, et fondent ensemble notre rapport au monde phénoménal (nous voyons le monde la plupart du temps en le reconnaissant, et de temps en temps en le constituant).

Cependant, le choix d'une esthétique de la vision particulière peut conduire un film documentaire à adopter tel ou tel mode d'introduction de ses objets. Si l'ébauche de notre analyse de Shoah permet d'admettre que certains films à l'intérieur de l'ensemble documentaire se rattachent à une esthétique de la constitution, Shoah en constituerait de la sorte son paradigme. Dans un texte de la revue Hors Cadre, Pierre Sorlin écrit: "Shoah ne participe en rien à la constitution d'une mémoire au sens où ce mot est pris ici, c'est-à-dire comme remémoration... il s'agit d'un monument " (1991, p. 29). Nous proposerons dès lors d'appeler monumentaires les films qui se rattachent à cette esthétique. Empruntant le terme à Pierre Sorlin, nous ne manquerons pas de songer à Michel Foucault, pour qui la monumentalisation d'un document consiste à lui redonner son contexte intrinsèque, sa consistance originelle.

\section{Sujets, objets, temporalité du monumentaire}

En vue de l'exploration des caractéristiques des films «monumentaires" et d'une esthétique de la constitution, nous tenterons d'abord de savoir quelle image du Sujet peut être proposée par ces films; ensuite, nous examinerons ce qui advient de ses objets. Enfin, nous interrogerons le Temps des monumentaires.

Dans un documentaire "classique", le Sujet du film, c'està-dire le Sujet construit par le film et accepté par le spectateur quand il y reconnaît un documentaire ${ }^{3}$, est d'abord le garant des actes de référence opérés par le film. Le Sujet est celui qui a vu, et qui a effectivement entendu les objets réels dont les images du 
film témoignent. Le Sujet est celui qui s'est immergé dans la configuration d'espace-temps présupposée par le film. C'est en cela qu'il est un Sujet réel (un "Énonciateur présupposé réel» d'après Roger Odin). Néanmoins, ce Sujet est aussi celui qui a organisé ses perceptions, qui les a distribuées dans le film, pour faire apparaître un certain niveau d'organisation des objets auxquels le film fait référence. De ce point de vue, le Sujet est aussi hors de la configuration, dans une position d'observateur extérieur. Dire que le spectateur comprend le film signifie qu'il discerne les passages du Sujet du Dedans au Dehors (et inversement), qu'il suture les sautes de perception issues de ces passages.

En général, le Sujet d'un monumentaire n'est pas présumé le garant réel d'une certaine configuration d'espace et de temps, puisque celle-ci n'est pas augurée par un tel film (quelques exceptions existent: les fillms où la configuration constituée par le film est le monde du Sujet du film, à l'instar de films de famille destinés à un public vaste comme Laurent et Stéphane de Patrick Gaudin, ou le récit autobiographique d'Hervé Guibert ${ }^{4}$ ). Un tel Sujet est d'abord celui qui dit: «il y a eu (quelque chose), ici, à tel moment." C'est donc lui qui constitue un lieu, et le place vis-à-vis d'autres configurations. Dans ses films les plus récents, et plus particulièrement dans Urgences, Raymond Depardon limite immédiatement, définitivement et précisément un lieu en disposant invariablement sa caméra dans une pièce. De ceux qui entrent ou sortent, nous ne saurons rien, sauf qu'ils sont contraints de passer par là. L'Arpentage ne réside pas dans la production de limites ou de frontières, mais plutôt dans la constitution d'un lieu à la fois clos et soumis aux pressions du Dehors. Quelle que soit la forme qu'il prenne, l'acte de production d'un lieu s'opère à l'intérieur d'un monde présupposé réel : c'est en cela qu'un monumentaire est une forme de documentaire, et que l'Arpenteur possède le statut de sujet réel. Du moins est-ce la proposition qui est faite au spectateur.

Cependant, l'Arpentage ne suffit pas: il faut encore organiser le "quelque chose" dont celui-ci a défini le lieu. Cette constitution émane de Sujets "de l'intérieur". Le Sujet de l'Arpentage doit s'effacer pour donner la parole à celui qui sait, parce qu'il est celui qui, à un moment ou à un autre, a vécu, senti, saisi. De 
la sorte, le lieu, jusque-là informe, se configure sous l'action de la parole; il s'organise, se caractérise, se constitue par et dans cette opération ici désignée Invocation. Les «Sujets de l'invocation" sont amenés à parler. Ils ont des traits individualisants. Qui a vu Shoah et pourra oublier Simon Srebnik ou Abraham Bomba? Qui a vu Urgences et pourra oublier cette femme liée, hurlante, toujours en mouvement? Pourtant, ils ont aussi des traits propres à l'Impersonnel ou au Collectif. Quand Simon Srebnik raconte la crémation des cadavres, ne parle-t-il pas au nom de tous ces cadavres, de tous ceux qui ont été brûlés? Et cette femme, qui veut retrouver son enfant, ne devient-elle pas la voix de tous ceux qui sont atteints par un tel déchirement? À propos de Shoah, Pierre Sorlin écrit: «... ce qui le distingue de tout ce qui était auparavant accessible est la douleur de la parole" (1991, p. 29). À force de s'enfoncer en elle-même, cette douleur devient une pure abstraction, une Priméité selon Peirce. En cela, elle devient l'expression d'un sentiment collectif.

Entre les Sujets qui composent le Sujet d'un monumentaire, l'Arpenteur et l'Invoquant, il y a la donation de la parole, de l'un à l'autre. Celui qui a tracé les limites s'efface pour abandonner la parole à celui qui se trouve — ou s'est trouvé - à l'intérieur de ces limites. Bien sûr, l'Arpenteur ne disparaît pas complètement quand il cède la place à l'Invoquant: il reste présent par son acte de donation, qui se poursuit tant que l'Invoquant conserve la parole. Cependant, ce dernier devient le garant de la constitution du lieu. Il porte sur ses épaules le mouvement configurant, et la crédibilité de ce mouvement. D'où vient, sans doute, l'impression qu'un monumentaire est, beaucoup moins qu'un documentaire classique, l'œuvre d'un "auteur»: le Sujet construit par le film y est moins individualisé et plus complexe. On n'est pas loin du paradoxe d'une énonciation à la fois subjective et impersonnelle.

De plus, la compréhension d'un monumentaire dépend d'un autre type de suture que le documentaire classique: le spectateur doit conjoindre un visible - qui précise les limites d'une topographie - et une parole - qui offre des visions. Suturer, ici, c'est saisir le balancement entre l'établissement d'une frontière et le visionnement d'un monde contenu dans une parole. 
L'objet du documentaire classique - ce à quoi il fait référence - est en général aisément spécifiable: il s'agit d'un ensemble d'objets réels appartenant à un monde présupposé réel. Encore une fois, cela ne veut pas pour autant dire que le discours sur ces objets soit forcément convenu. Il suffit d'évoquer $A$ propos de Nice pour éviter une telle conclusion, fort hâtive. Le statut que le monumentaire accorde à son objet est plus difficile à définir. Pour reprendre nos conclusions sur la référenciation dans Shoah, nous proposions que celle-ci s'apparente à ce que Strawson appelle l'introduction de concepts-traits dans des énoncés du type: "il y a de l'or, de la neige, $d u$ chat..." De tels énoncés contiennent l'idée de la présence de configurations d'espace et de temps particulières.

Nous voudrions nous permettre d'interpréter les notions de concepts-traits et d'énoncés qui-placent-des-traits. Pour cela, nous rapprocherons, peut-être un peu abruptement, quelques-unes des remarques de Strawson et plusieurs définitions du philosophe américain $A$. N. Whitehead. Ce dernier fonde son approche des phénomènes sur les concepts d'événement et d'espace-temps. Un événement est déterminé par le caractère particulier de ce qui apparaît à quelqu'un: "What we discern is the specific character of a place through a period of time. This is what I mean by an "event" (Whitehead, 1920, p. 52). De plus, un espace-temps est "la spécification de certaines qualités générales des événements" (Whitehead, 1930, p. 92); il est la part abstraite de l'événement pour l'esprit humain qui nomme et décrit. L'événement et l'espace-temps sont toujours associés à un ou plusieurs sujets.

De la sorte, il me semble qu'il y ait dans un énoncé qui-placeun-trait la formulation de ce qui fait l'unité d'un événement. "Il y a du chat» signifierait la perception d'un lieu organisé par le passage d'un chat, ou autour de ce passage. Un tel énoncé marquerait à la fois l'unité de cette perception, et le principe de son organisation. Le concept-trait lui même renverrait à l'espacetemps induit de l'expérience constitué par ce passage d'un chat (nous avons vu que Strawson lui-même montre que de tels concepts présupposent des configurations d'espace-temps).

En toute hypothèse, nous poserons que les objets des monumentaires sont des événements, contrairement au documentaire 
classique où ces objets sont des objets réels, des choses, des corps. (Ce n'est pas ici le lieu de développer l'opposition entre les «événements» et les «choses». Nous espérons que l'usage courant, et les quelques lignes qui précèdent suffiront.) Cette opposition ne se confond pas avec celle qui distingue les particuliers et les universaux: les événements sont individualisés au même titre que les objets réels. Chaque événement est unique et bien spécifié, comme "cette table" sur laquelle nous écrivons en ce moment. Néanmoins, la singularité d'un événement n’est pas du même ordre que celle de l'objet réel : elle est d'abord historique. Un événement s'inscrit dans des limites spatiales et temporelles. De plus, il se définit par la forme donnée à la portion d'espace et de temps qu'il érige. Décrire un événement ne peut juste décliner l'ensemble des objets réels qui sont les traces de l'événement. Il faut plutôt dépeindre l'incurvation de l'espace et du temps que représente cet événement.

Dire qu'un monumentaire fait référence à un événement, c'est donc penser qu'il s'attache à décrire le caractère spécifique d'un fait humain : ce qui est arrivé, dans tel lieu et à tel moment, à telle ou telle personne. Il lui faut constituer le jeu de langage de cet événement, ses présupposés, ses règles, ses croyances. Mais ceci n'est possible - sous réserve d'accepter la définition de l'événement donnée par Whitehead - qu'en s'effaçant devant ce dernier. Pour être un monumentaire, le Sujet principal, l'Arpenteur doit accepter d'être dessaisi du discours du film. Plus exactement, il doit lui-même construire ce dessaisissement, et ouvrir, au cœur même du film, un lieu silencieux qui puisse accueillir la parole de l'Invoquant, c'est-à-dire la parole du Sujet de l'événement. Un monumentaire est un film à deux voix, dont la première s'attache à aider la seconde à désigner la référence du film.

Déclinant les qualités d'un événement, le monumentaire contribue à en établir la forme générale: comme l'architecture formelle qui est à la fois spatiale et temporelle, il compose l'espacetemps de l'événement où prend place l'événement. Il ne se confond donc pas avec lui, mais en condense l'organisation et l'agencement. La considération des espaces-temps est ainsi un moyen d'opposer ou de trouver semblables des événements 
différents. Ainsi, Shoah parât fournir des indications pour comparer certaines purifications ethniques et la Solution finale.

Cette remarque nous amène à quelques considérations relatives à la temporalité. Il semble en effet que le documentaire classique entretient généralement des rapports privilégiés avec la chronologie, dans la mesure où les objets réels auxquels il est fait référence doivent être situés dans le temps linéaire, c'est-à-dire le temps de l'existence physique.

Le Temps du monumentaire est d'abord celui du présent de l'événement. Ce dernier n'a de sens que par son inscription dans un temps historique, humain, à l'intérieur duquel il fait rupture: d'une part, il désigne son passé, et, d'autre part, il annonce son futur. Un monumentaire construit ainsi sa conception du temps, son interprétation du temps: il reconstruit une temporalité à partir de l'événement, qui est sa référence. Ce qui dans l'événement échappe au présent, pour régir une telle recomposition. Shoah traverse l'histoire de tous les camps, sans omettre d'extraire de notre propre histoire tous ces massacres, en Bosnie, au Rwanda, ou en Tchétchénie, que nous traversons avec pas moins d'indifférence que les paysans polonais de Chelmno. Dans Urgences, la parole qui souffre et la parole qui contrôle façonnent une figure déviante de la socialité, qui est comme une menace au-dessus de notre société particulièrement institutionnalisée. Tout monumentaire serait une proposition temporelle, une figure ou une image du temps historique. Il vise donc l'espace public, ou plutôt il en appelle à l'espace public. En produisant une proposition existentielle et en donnant une version du temps, il réclame un jugement. L'esthétique n'est rien, ici, sans l'éthique.

\section{Conclusion}

Si les monumentaires sont assez peu nombreux, sans doute est-ce parce qu'ils exigent une part d'ascèse des auteurs et des spectateurs. Il s'agit en effet d'oublier les garanties qu'offrent les mondes reconnus, bien balisés, pour entrer dans le domaine de l'autre. Chaque monumentaire s'apparente à un acte de croyance qui affirme une possible réconciliation avec le vécu de cet autre. Dans L'Image-temps, Gilles Deleuze constate que... 
le fait moderne, c'est que nous ne croyons plus en ce monde... Seule la croyance au monde peut [aujourd'hui] relier l'homme à ce qu'il voit et entend. Il faut que le cinéma filme, non pas le monde, mais la croyance à ce monde notre seul lien" (1985, p. 223).

Si le cinéma doit obéir à certains devoirs, le monumentaire ne doit en aucune manière craindre de l'assumer. L'éthique, qui n'a décidément rien d'inconvenant, mérite un égard certain dès qu'il est question de cinéma.

\section{Université de Lyon 3}

\section{NOTES}

1. Nous reprenons sur ce point une position empruntée à Ducrot, par exemple dans Les mots du discours (1980, p. 33-56).

2. Gilles Deleuze, conférence à la FEMIS, enregistrement personnel (1987).

3. Notons, en passant, et bien que ne soit pas ici notre sujet, que cette acceptation repose sur le "savoir de l'arché": le spectateur s'appuie sur son savoir concernant la technique cinématographique pour accepter de reconnaître les images et les sons du film comme des empreintes de la réalité.

4. Sur ce type de film, voir notre article "L'effet "film de famille" (Esquenazi, 1995, p. 217-224).

\section{RÉFÉRENCES BIBLIOGRAPHIQUES}

Deleuze, 1985: Gilles Deleuze, L'Image-temps, Paris, Minuit, 1985.

Ducrot, 1980 : Oswald Ducrot, Les Mots du discours, Paris, Minuit, 1980.

Esquenazi, 1994: Jean-Pierre Esquenazi, Film, perception et mémoire, Paris, L'Harmattan, 1994.

Esquenazi, 1995: Jean-Pierre Esquenazi, "L'effet "film de famille" ", dans Roger Odin (dir.), Le Film de famille, Paris, Méridiens Klincksieck, 1995, p. 207-224.

Odin, 1984: Roger Odin, "Film documentaire, lecture documentarisante", dans Jean-Charles Lyant et Roger Odin (dir.), Cinémas et réalités, Saint-Étienne, CIEREC, 1984, p. 263-278.

Sorlin, 1991 : Pierre Sorlin, "Une mémoire sans souvenir ", Hors Cadre, n 9, 1991.

Strawson, 1973: Peter Frederick Strawson, Les Individus, Paris, Seuil, [1959] 1973.

Whitehead, 1920: Alfred North Whitehead, The Concept of Nature, Cambridge, Cambridge University Press, 1920.

Whitehead, 1930 : Alfred North Whitehead, La Science et le monde moderne, Paris, Payot, [1925] 1930. 